\title{
Quantum search by partial adiabatic evolution
}

\author{
Ying-Yu Zhang* and Song-Feng Lu \\ School of Computer Science, Huazhong University of Science and Technology, Wuhan, China.
}

(Dated: December 27, 2017)

\begin{abstract}
A quantum search algorithm based on the partial adiabatic evolution [1] is provided. We calculate its time complexity by studying the Hamiltonian in a two-dimensional Hilbert space. It is found that the algorithm improves the time complexity, which is $O(\sqrt{N / M})$, of the local adiabatic search algorithm[2], to $O(\sqrt{N} / M)$.

PACS numbers: 03.67.Lx
\end{abstract}

\section{INTRODUCTION}

Quantum adiabatic computation has attracted a lot of attention in the past decades, such as [1, 3-10], since it was proposed by Farhi et al.[11]. In [5], an adiabatic algorithm was proposed to solve the Deutsch-Jozsa problem. The algorithm took an exponential time which provided only a quadratic speed up over the best classical algorithm for the problem. A modified algorithm proposed by Wei and Ying 12] improved the performance to constant time. In [6], quantum adiabatic computation was proved to be polynomially equivalent to the quantum circuit model. The proof showed that adiabatic quantum computation using Hamiltonians with long-range fiveor three-body interactions, or nearest-neighbor two-body interactions with six-state particles, could efficiently simulate the circuit model. This results was soon modified to qubits with two-body interactions [13]. A simpler proof of the Equivalence was presented in [8]. In [14], quantum adiabatic computation was applied to solve random instances of NP-complete problems. A research outline of its application to solve NP-complete problems can also be found in the paper.

A typical quantum adiabatic algorithm starts with the ground state of the initial Hamiltonian $H_{i}$, and evolves slowly to the ground state of the final Hamiltonian $H_{f}$. The system that implements the algorithm uses the timedependent Hamiltonian

$$
H(s(t))=(1-s(t)) H_{i}+s(t) H_{f} .
$$

The running time (evolution time) is essentially determined by [1]

$$
T \geq \Theta\left(\frac{1}{g_{\min }^{2}}\right),
$$

where

$$
g_{\min }=\min [E(1, s)-E(0, s)],(0 \leq s \leq 1) .
$$

$E(0, s)$ and $E(1, s)$ are the two lowest eigen values of $H(s)$. Roland and Cerf [2] considered the unstructured

\footnotetext{
* newpine2002@yahoo.com.cn
}

search problem [15], and designed a quantum search algorithm based on local adiabatic evolution. In the algorithm, $H_{i}$ and $H_{f}$ are specified as

$$
H_{i}=1-|\Psi\rangle\langle\Psi|
$$

and

$$
H_{f}=1-|\beta\rangle\langle\beta|
$$

where

$$
|\Psi\rangle=\frac{1}{\sqrt{N}} \sum_{i=0}^{N-1}|i\rangle
$$

and $|\beta\rangle$ is an equal superposition of all marked states. The algorithm can find one marked item in a running time of order $\sqrt{N / M}$.

In [1], Tulsi proposed a partial adiabatic evolution with $H_{f}$ a one-dimensional projector Hamiltonian. It was also checked in the paper that Roland and Cerf's results (of the case that there is only one marked state) can be obtained as a special case of the partial adiabatic evolution. In this paper, we give a specified quantum search algorithm based on the partial adiabatic evolution, and show that the algorithm provides a better time complexity, which is $O(\sqrt{N} / M)$, than that, which is $O(\sqrt{N / M})$, of the local adiabatic search algorithm.

This paper is organized as follows. In section 2 , we specify the partial adiabatic search algorithm. In section 3 , we calculate its time complexity. We conclude the paper in section 4 .

\section{PARTIAL ADIABATIC SEARCH ALGORITHM}

In this section, we specify the partial adiabatic evolution [1] as a search algorithm. For convenience, we use different notations. The search algorithm executes the four steps below:

(1) The initial state is prepared to be $|\Psi\rangle$.

(2) At $\mathrm{t}=0$, the Hamiltonian is suddenly changed to $H\left(s^{-}\right)$without disturbing the state $|\Psi\rangle$.

(3) The Hamiltonian evolves from $H\left(s^{-}\right)$to $H\left(s^{+}\right)$linearly in time over duration $T^{\prime}$. 
(4) Measure the state of the system.

Repeat these four steps until we find a marked state. For the algorithm, $s^{-}$and $s^{+}$are specified as $s^{-}=\frac{1}{2}-$ $\frac{1}{2 \sqrt{N}}$ and $s^{+}=\frac{1}{2}+\frac{1}{2 \sqrt{N}}$. Because the algorithm evolves adiabatically only within a small time interval $\left[s^{-}, s^{+}\right]$, it is called a partial adiabatic search algorithm. After step 2 , the system that implements the algorithm will be still in the state $|\Psi\rangle$. That is, the system state will be the ground state of $H\left(s^{-}\right)$with probability $\left|\left\langle\Psi \mid E\left(0, s^{-}\right)\right\rangle\right|^{2}$. The adiabatic theorem [16] guarantees that it will be the ground state of $H\left(s^{+}\right)$with probability $\left|\left\langle\Psi \mid E\left(0, s^{-}\right)\right\rangle\right|^{2}$ after step 3. Measuring the state of the system will give the ground state of $H_{f}=1-|\beta\rangle\langle\beta|$ with probability $P=\left|\left\langle\Psi \mid E\left(0, s^{-}\right)\right\rangle\right|^{2} \times\left|\left\langle\beta \mid E\left(0, s^{+}\right)\right\rangle\right|^{2}$. We call $\mathrm{P}$ oneround success probability, and accordingly $T^{\prime}$ one-round evolution time. The overall time complexity(evolution time) of the algorithm is $T=T^{\prime} / P$. Here, $T^{\prime}=\omega / g_{\text {min }}^{2}$, where $\omega=s^{+}-s^{-}=\frac{1}{\sqrt{N}}$.

\section{TIME COMPLEXITY}

\section{A. minimum energy gap}

Let

$$
\begin{array}{r}
|\alpha\rangle=\frac{1}{\sqrt{N-M}} \sum_{x \notin S}|x\rangle, \\
|\beta\rangle=\frac{1}{\sqrt{M}} \sum_{x \in S}|x\rangle,
\end{array}
$$

where $\mathrm{S}$ is the set of the marked states, and $\mathrm{M}$ is the number of the marked states. Throughout of this paper, we suppose $\mathrm{S}$ is not empty. This means that $1 \leq M \leq N$. The state $|\Psi\rangle$ can be rewritten as 17.

$$
|\Psi\rangle=\sqrt{\frac{N-M}{N}}|\alpha\rangle+\sqrt{\frac{M}{N}}|\beta\rangle .
$$

It is easily to check that any state orthogonal to $|\alpha\rangle$ and $|\beta\rangle$ is a eigen state of $H(s)$ and the corresponding eigen value is 1 which is $(\mathrm{N}-2)$ times degenerated. This means that $E(k, s)(k=0,1)$ and are in the subspace spanned by $|\alpha\rangle$ and $|\beta\rangle$. Besides, all the following calculations involve states only in the two-dimensional subspaces panned by $|\alpha\rangle$ and $|\beta\rangle$. So, we can work in the subspace, instead of working in the $\mathrm{N}$-dimensional Hilbert space.

Let the eigen spectrum of $\mathrm{H}(\mathrm{s})$ be

$$
H(s)|E(k, s)\rangle=E(k, s)|E(k, s)\rangle
$$

where $E(k, s)$ and $|E(k, s)\rangle$ are the k-level eigen value and eigen state of $H(s)$ respectively. Throughout this paper, we only consider the two lowest eigenstates and eigenvalues, i.e. $\mathrm{k}=0,1$. Left multiplying $\langle\alpha|$ to Eq.(10), we get

$$
\langle\alpha|H(s)| E(k, s)\rangle=E(k, s)\langle\alpha \mid E(k, s)\rangle .
$$

Substituting the Eq.(1) into the left side of Eq.(11), we also get

$$
\begin{array}{r}
\langle\alpha|H(s)| E(k, s)\rangle= \\
\langle\alpha \mid E(k, s)\rangle-(1-s)\langle\alpha \mid \Psi\rangle\langle\Psi \mid E(k, s)\rangle,
\end{array}
$$

with $\langle\alpha \mid \beta\rangle=0$. Combining Eqs. (11) with (12), we obtain

$$
\langle\alpha \mid E(k, s)\rangle=\frac{(1-s)\langle\alpha \mid \Psi\rangle\langle\Psi \mid E(k, s)\rangle}{1-E(k, s)},
$$

when $1-E(k, s) \neq 0$. Similarly,

$$
\langle\beta \mid E(k, s)\rangle=\frac{(1-s)\langle\beta \mid \Psi\rangle\langle\Psi \mid E(k, s)\rangle}{1-s-E(k, s)},
$$

when $1-s-E(k, s) \neq 0$. Thus, with Eqs.(13) and (14), we get

$$
\begin{array}{r}
\langle\Psi \mid E(k, s)\rangle=\langle\Psi \mid \alpha\rangle\langle\alpha \mid E(k, s)\rangle+\langle\Psi \mid \beta\rangle\langle\beta \mid E(k, s)\rangle \\
=\left(\frac{(1-s) A}{1-E(k, s)}+\frac{(1-s) B}{1-s-E(k, s)}\right)\langle\Psi \mid E(k, s)\rangle(
\end{array}
$$

where $A=|\langle\Psi \mid \alpha\rangle|^{2}=\frac{N-M}{N}$ and $B=|\langle\Psi \mid \beta\rangle|^{2}=\frac{M}{N}$. Rearranging Eq.(15), we finally obtain the secular function for $\mathrm{H}(\mathrm{s})$

$$
E^{2}(k, s)-E(k, s)+s(1-s) A=0 .
$$

So,

$$
E(0, s), E(1, s)=\frac{1 \pm \sqrt{1-4 s(1-s) A}}{2}
$$

and

$$
g_{s}=E(1, s)-E(0, s)=\sqrt{1-4 s(1-s) A} .
$$

The minimum energy gap $g_{\min }=\sqrt{1-A}=\sqrt{M / N}$ is obtained for $s=1 / 2$. The one-round evolution time is $T^{\prime}=\sqrt{N} / M$.

\section{B. one-round success probability}

In this subsection, we calculate the one-round success probability. Substituting Eqs.(13) and (14) into $|\langle\alpha \mid E(k, s)\rangle|^{2}+|\langle\beta \mid E(k, s)\rangle|^{2}=1(k=0,1)$, we have

$$
\left(\frac{(1-s)^{2} A}{(1-E(k, s))^{2}}+\frac{(1-s)^{2} B}{(1-s-E(k, s))^{2}}\right)|\langle\Psi \mid E(k, s)\rangle|^{2}=1 \text {. }
$$

when $1-E(k, s) \neq 0$ and $1-s-E(k, s) \neq 0$. If $1-s \neq 0$, this immediately gives

$$
|\langle\Psi \mid E(k, s)\rangle|^{2}=\frac{1}{(1-s)^{2}\left(\frac{A}{(1-E(k, s))^{2}}+\frac{B}{(1-s-E(k, s))^{2}}\right)} .
$$

Substituting Eq.(20) into Eq.(14), we get

$$
|\langle\beta \mid E(k, s)\rangle|^{2}=\frac{B}{\left(\frac{(1-s-E(k, s))}{1-E(k, s)}\right)^{2} A+B} .
$$


Because

$$
\begin{gathered}
\left(1-s^{-}\right)^{2}=\frac{1}{4}\left(1+\frac{1}{\sqrt{N}}\right)^{2}<1, \\
\frac{A}{\left(1-E\left(0, s^{-}\right)\right)^{2}}=\frac{4(N-M) / N}{\left(1+\sqrt{\frac{1+M}{N}-\frac{M}{N^{2}}}\right)^{2}}<4
\end{gathered}
$$

and

$$
\begin{array}{r}
\frac{B}{\left(1-s^{-}-E\left(0, s^{-}\right)\right)^{2}}=\frac{4 M}{\left(1+\sqrt{1+M-\frac{M}{N}}\right)^{2}} \\
\leq \frac{4 M}{(1+\sqrt{M})^{2}}<4
\end{array}
$$

we obtain

$$
\left|\left\langle\Psi \mid E\left(0, s^{-}\right)\right\rangle\right|^{2}>\frac{1}{8} .
$$

using Eq. 20.

Because

$$
\begin{array}{r}
\frac{1-s^{+}-E\left(0, s^{+}\right)}{1-E\left(0, s^{+}\right)}=\frac{\frac{-1}{\sqrt{N}}+\sqrt{\frac{M+1}{N}-\frac{M}{N^{2}}}}{1+\sqrt{\frac{M+1}{N}-\frac{M}{N^{2}}}} \\
<\frac{-1}{\sqrt{N}}+\sqrt{\frac{M+1}{N}-\frac{M}{N^{2}}}<\sqrt{\frac{2 M}{N}}=\sqrt{2 B},
\end{array}
$$

we obtain

$$
\left|\left\langle\beta \mid E\left(0, s^{+}\right)\right\rangle\right|^{2}>\frac{B}{2 B A+B}=\frac{1}{2 A+1}>\frac{1}{3},
$$

using Eq. 21.

This provides a lower bound of the one-round success probability

$$
P=\left|\left\langle\Psi \mid E\left(0, \frac{1}{2}-\frac{1}{2 \sqrt{N}}\right)\right\rangle\right|^{2} \times\left|\left\langle\beta \mid E\left(0, \frac{1}{2}+\frac{1}{2 \sqrt{N}}\right)\right\rangle\right|^{2}>\frac{1}{24} .
$$

So, the overall time complexity of the partial adiabatic search algorithm is $T=T^{\prime} / P=O(\sqrt{N} / M)$.

\section{CONCLUSION}

We have provided a quantum search algorithm based on the partial adiabatic evolution. As we have seen that the minimum energy gap along with the one-round success probability determine the overall time complexity of the algorithm. They are calculated by studying the time-dependent Hamiltonian $H(s)$ in a two-dimensional Hilbert space. The overall time complexity is $O(\sqrt{N} / M)$, which provides a speed up of $\sqrt{M}$ over the local adiabatic search algorithm. This is resulted from the fact that the one-round success probability is bounded from below by a constant.
[1] A. Tulsi, Physical Review A 80, 052328 (2009).

[2] J. Roland and N. J. Cerf, Physical Review A 65, 042308 (2002).

[3] A. M. Childs, E. Farhi, and J. Preskill, Physical Review A 65, 012322 (2001).

[4] E. Farhi, J. Goldstone, S. Gutmann, J. Lapan, A. Lundgren, and D. Preda, Science 292, 472 (2001).

[5] S. Das, R. Kobes, and G. Kunstatter, Physical Review A 65, 062310 (2002).

[6] D. Aharonov, W. van Dam, J. Kempe, Z. Landau, S. Lloyd, and O. Regev, Proceedings of the 45th Annual IEEE Symposium on Foundations of Computer Science(FOCS'04)(2004).

[7] Z. Wei and M. Ying, Physical Review A 76, 024304 (2007).
[8] A. Mizel, D. A. Lidar, and M. Mitchell, Physical Review Letters 99, 070502 (2007).

[9] E. Farhi, J. Goldstone, D. Gosset, S. Gutmann, H. B. Meyer, and P. Shor, quant-ph/0909.4766v2.

[10] T. Jörg, F. Krazkala, G. Semerjian, and F. Zamponi, Physical Review Letters 104, 207206 (2010).

[11] E. Farhi, J. Goldstone, S. Gutmann, and M. Sipser, arXiv:quant-ph/0001106v1(2000).

[12] Z. Wei and M. Ying, Physics Letters A 354, 271 (2006).

[13] S. A. Chin and E. Krotscheck, Physical Review A 71, 062314 (2005).

[14] B. Altshuler, H. Krovi, and J. Roland, quant$\mathrm{ph} / 0908.2782 \mathrm{v} 2$.

[15] L. K. Grover, Physical Review Letters 79, 325 (1997).

[16] A. Messiah, Quantum Mechanics (Dover, 1999).

[17] M. A. Nielsen and I. L. Chuang, Quantum Computation and Quantum Information (Cambridge University Press, 2000). 